\title{
El Enfoque de Competencias en la Formación Universitaria y su Impacto en la Evaluación. La Perspectiva de un Grupo de Profesionales Expertos en Pedagogía
}

\author{
Carmen López ${ }^{(1)}$, Vicente Benedito(2) y María J. León ${ }^{(1)}$ \\ (1) Universidad de Granada, Facultad de Ciencias de la Educación, Campus Universitario Cartuja s/n, \\ 18011, Granada, España. (e-mail: mclopez@ugr.es, mleon@ugr.es) \\ (2) Universidad de Barcelona. Facultad de Educación. Universidad de Barcelona. Campus Vall d'Hebron, \\ Edifici de Migdia, Passeig de la Vall d'Hebron, 171, 08035, Barcelona, España. (e-mail: vbenedito@ub.edu)
}

Recibido Dic. 11, 2015; Aceptado Ene. 27, 2016; Versión final Mar. 19, 2016, Publicado Ago. 2016

\begin{abstract}
Resumen
El artículo profundiza en las reflexiones y valoraciones realizadas por un grupo de personas expertas en Pedagogía de siete universidades españolas sobre el enfoque de competencias y su impacto en la evaluación y mejora de la formación universitaria. Se trata de una investigación narrativa centrada en el análisis de las respuestas proporcionadas por los participantes a través de un cuestionario de preguntas abiertas. Los resultados evidencian que la incorporación del enfoque de competencias a la formación universitaria representa una oportunidad para ensalzar el valor formativo de la práctica. Sin embargo, su adopción puede suponer una vuelta a posicionamientos tecnológicos ampliamente cuestionados y una subordinación a las demandas del mercado. Para evitarlo, el panel de expertos propone mejorar las condiciones en las que se ejerce la enseñanza, prestar mayor atención a la formación del profesorado, y ampliar la reflexión y debate en torno al enfoque de competencias y a los sistemas de evaluación.
\end{abstract}

Palabras clave: educación superior; enfoque de competencias; evaluación; investigación narrativa; pedagogos expertos

\section{The Approach by Competency in University Training and its Impact on Student Evaluation. The Perspective of a Team of Pedagogy Experts}

\begin{abstract}
The present paper examines the reflections and opinions provided by a pedagogy expert team from six Spanish Universities on competency-based training and its impact on assessment procedures and on improvements in University teaching and learning processes. This is a narrative inquiry which focuses on the analysis of answers provided by participants through a survey, employing open-ended questions. The results provide evidence to suggest that the adoption of a competency-based approach may be a good opportunity to highlight the value of practical training components. However, experts are concerned that implementing such an approach may represent a return to more technical forms of training, which are somewhat questionable and show subordination to market demands. To avoid this, the experts propose improving the conditions in which the education is developed, focusing the attention on the teacher-training, and a widening the debate around competency-based training and on the assessment systems.
\end{abstract}

Keywords: higher education; competency-based approach; assessment; narrative inquiry; expert teachers 


\section{INTRODUCCIÓN}

El nuevo escenario social, globalizado y postindustrial, requiere de una redefinición y reconstrucción del sentido y finalidad de la institución universitaria. En el marco europeo, este proceso se inicia con la Declaración de La Sorbona en mayo de 1998, en la que se reivindica una Europa del conocimiento que permita acabar con las fronteras educativas y desarrollar un marco de enseñanza y aprendizaje que favorezca la movilidad y cooperación en el seno de la Unión. Un año más tarde, con la Declaración de Bolonia, se ratifican los acuerdos adoptados en La Sorbona y se sientan las bases para crear el Espacio Europeo de Educación Superior (EEES), proceso que culminaría en 2010 y que se conoce como Proceso de Bolonia. En este proceso se apuesta por la Europa del conocimiento como factor clave para el desarrollo social y humano de la ciudadanía.

Estos acuerdos se han concretado en la incorporación de un enfoque educativo centrado en el aprendizaje de competencias. En opinión de Coll (2007), su adopción representa un enriquecimiento en la medida en que supone integrar y movilizar distintos tipos de aprendizajes (conocimientos, habilidades y actitudes) para afrontar situaciones y problemas en contextos determinados. Favorece así, aprender autónomamente, autorregular y dirigir el propio aprendizaje y continuar aprendiendo a lo largo de la vida. Este potencial formativo conferido a las competencias viene acompañado de un mayor protagonismo del estudiante en sus procesos de formación y de un cambio significativo en las propuestas formativas y en la propia cultura docente universitaria. Para López (2011), el enfoque por competencias invita al establecimiento de compromisos conjuntos en todo lo relativo al diseño, desarrollo y evaluación curricular, incorporando nuevas claves en la docencia universitaria.

El carácter integrador, transferible y multifuncional de las competencias requiere diseños y desarrollos curriculares más coordinados e interdisciplinares, que posibiliten una educación más integral, la incorporación de metodologías activas y tareas con las que el alumnado aprenda a afrontar situaciones complejas y una nueva filosofía en torno a la evaluación que la convierta en una práctica verdaderamente educativa. Como han señalado Marín et al. (2013: 49), "la evaluación de competencias proporciona la oportunidad de transformar la evaluación como control, en evaluación como mejora; y de concebirla como un momento más de aprender".

Sin embargo, una educación superior orientada a la adquisición de competencias no está exenta de polémica (Bolívar, 2011). El diseño de nuevos planes de estudios sustentados en perfiles profesionales, la relevancia conferida a los logros, los nuevos roles docentes y su escasa formación, así como la trascendencia del discurso competencial y su impacto en la formación universitaria, son algunas de las cuestiones más debatidas y controvertidas y, por ello, se convierten en ejes centrales de este trabajo.

En opinión de Escudero (2009), la ola reformista que invade la institución universitaria responde a dos lógicas bien diferenciadas. "Una de ellas discurre por un mayor reconocimiento del papel y el valor de la educación en la sociedad del conocimiento y busca reconstruir los sistemas educativos de forma radical, con el objetivo de garantizar a todas las personas una educación de mayor calidad, más justa y equitativa al mismo tiempo" (Escudero, 2009: 67). Desde esta postura, se aboga por una educación humanista, democrática y capaz de impulsar la cohesión social. "La otra, en la que también se reconoce el valor estratégico de la educación en la economía del conocimiento, lo que se busca es la excelencia, el sometimiento de la formación a los imperativos de la globalización económica y las demandas del mercado, la innovación y la competitividad mercantil, la racionalización, eficiencia y eficacia de los sistema escolares en todos sus niveles, el aprendizaje a lo largo de toda la vida" (Escudero, 2009: 67). Se trata de una posición, esta última, influida por el neoliberalismo que enfatiza más el valor de la formación para el trabajo, la empleabilidad y el emprendimiento.

En los últimos tiempos han sido considerables los esfuerzos realizados para acometer la organización de la nueva oferta formativa, pero muy poca la atención dispensada al cambio cultural y cualitativo que representa, para la universidad y los docentes, la incorporación del nuevo enfoque educativo. La escasa atención conferida a esta reconversión cualitativa y el olvido sistemático deparado al profesorado son, como han reconocido Rué y Lodeiro (2010) y López (2011), serios obstáculos para la consolidación y el éxito del cambio que se persigue.

Sería un error desconsiderar que la formación universitaria en España ha estado marcada por la supremacía del modelo académico de formación frente al modelo profesionalizante. Este hecho ha generado y perpetuado importantes brechas entre el enfoque disciplinar y humanista de la formación universitaria y el encaminado a la formación de profesionales. El primero, más centrado en el "saber", ha gozado de elevado prestigio y reconocimiento social, mientras que la formación profesional, más interesada en impulsar el "saber hacer", no ha disfrutado del mismo status. Para Tejada (2006), esta división entre el mundo académico y el laboral encierra prejuicios y tabúes sociales, al tiempo que lleva a ciertos equívocos al apuntar que la universidad no profesionaliza. Más allá de esta apreciación, que estimamos acertada, tenemos que admitir que el 
desencuentro entre ambos posicionamientos ha sido uno de los rasgos que ha caracterizado la formación universitaria.

Esta escisión entre el mundo universitario y el laboral, denunciada por Jackson (2012) y ampliamente arraigada en las mentes y prácticas formativas, guarda estrecha relación con los temores generados con la adopción del enfoque competencial en la educación superior. En opinión de Bolívar (2011), este temor está justificado si se tiene en cuenta su origen, ligado al contexto empresarial y productivo, al enfoque taylorista y a procesos educativos cercanos al modelo de objetivos, preocupados, todos, por enfatizar el valor de los resultados de aprendizaje. "Su procedencia del mundo empresarial y profesional lo hacen sospechoso al vincularlo a las políticas neoliberales que subordinan la educación a las demandas del mercado" (Bolívar, 2011: 122).

Por otra parte, las fuertes críticas vertidas sobre la formación universitaria inciden en demandar más atención al contexto social, un mayor compromiso con la formación práctica y la necesidad de poner en marcha procesos formativos en los que se revalorice la práctica profesional. Abrir la puerta a nuevas metodologías basadas en la resolución de problemas, el trabajo por proyectos, aprendizaje cooperativo o estudios de casos, no sólo estrechan la conexión con la realidad profesional y el desarrollo de competencias (Halbaut et al., 2015), sino que ayudan a revalorizar la formación práctica.

La división de la realidad social en que se ha sustentado la formación universitaria (mundo laboral vs mundo académico) es hoy cuestionada e insostenible, y constituye uno de los aspectos clave desde los que se persigue redefinir el nuevo papel de las universidades. La creciente complejidad de los campos del saber y de los fenómenos sociales hace recomendable la adopción de perspectivas más sistémicas, integradoras y holísticas que subrayen el valor de las interdependencias y la complementariedad (Morin, 2000). Conviene, pues, huir de los posicionamientos que abordan la formación universitaria y el discurso de las competencias en términos antagónicos. Como ha señalado López (2011: 163), opinión que compartimos: "El enfoque de competencias no implica, necesariamente y de manera exclusiva, la adopción de una orientación mercantilista y neocapitalista", pero tampoco que debamos olvidar sus peligros y minimizar los riesgos que comporta (Barnett, 2001). Cabría pensar, entonces, que el interés por impulsar el valor de la práctica y el carácter profesionalizante de la formación universitaria no es el problema, aunque sí lo es el hecho de que la formación universitaria quede reducida exclusivamente a una formación para el empleo y se olvide o devalúe la formación humanista e intelectual.

A esta cuestión se une otra, no menos relevante e igualmente controvertida, que es la falta de formación docente del profesorado universitario (Parris y Saville, 2011). Este déficit no sólo dificulta el compromiso del colectivo docente con el cambio, sino que sitúa al profesorado universitario en una posición de debilidad desde la que resulta complejo hacer frente a los desafíos y dilemas que conlleva el enfoque de competencias y su traslación a la práctica educativa. En este sentido, conviene replantearse y valorar, por ejemplo, los términos en que se plantea el enfoque de competencias, pues la calidad del aprendizaje y el nivel de logro de cualquier competencia se apoyan, necesariamente, en apreciaciones subjetivas (Jornet et al. 2011) que requieren de un replanteamiento de los procesos de evaluación, algo que, por el momento, continua siendo una loable aspiración en la docencia universitaria (Ion y Cano, 2012).

Por otro lado, son numerosas las contribuciones que consideran a la evaluación uno de los aspectos clave del enfoque de competencias y demandan un cambio en los procedimientos utilizados en la educación superior. Brown y Glasner (2010), conscientes de las limitaciones del modelo de evaluación universitario y de la necesidad de abordar las distintas categorías de contenidos presentes en las competencias (conocimientos, habilidades,...), proponen un cambio que incremente el tiempo destinado a la orientación, seguimiento y feeback, al tiempo que aconsejan experimentar con otras opciones evaluadoras. En este sentido, estamos de acuerdo con López (2011) cuando señala que las claves sobre las que debe apoyarse la evaluación en la educación universitaria en estos momentos son tres: un análisis serio y riguroso que permita tomar conciencia de las fortalezas y debilidades del sistema de evaluación vigente, la adopción de una nueva lógica que ayude a redefinir el sentido y finalidad de la evaluación desde una perspectiva compleja e integral y, por último, la colaboración.

No cabe duda que ampliar los espacios para la reflexión y el debate, valorar los progresos alcanzados, las actuaciones emprendidas y compartir las inquietudes que genera el enfoque de competencias, es importante para vencer resistencias, prevenir riesgos y contribuir a la mejora de la práctica formativa. Algunos trabajos han analizado las perspectivas del profesorado, alumnado o empleadores respecto a estas cuestiones (Baartman et al. 2007; Palmer et al. 2009; Agudo et al. 2013). En este trabajo, en cambio, hemos querido acercarnos a los discursos del personal experto en Pedagogía para conocer su opinión sobre el enfoque de competencias y su relevancia e impacto en la enseñanza universitaria. La iniciativa se enmarca en un proyecto de investigación de mayor envergadura titulado: "El impacto de la evaluación educativa en el desarrollo de 
competencias en la universidad. La perspectiva de las primeras promociones de graduados" (Ref: EDU201232766), que ha sido financiado por el Ministerio de Economía y Competitividad de España.

El propósito del estudio presentado en este trabajo es recabar información sobre el enfoque de competencias adoptado en la educación universitaria, su contribución a la calidad de la docencia y la formación universitaria, y su impacto en la evaluación de los aprendizajes, desde la perspectiva de un grupo de personas expertas en Pedagogía.

\section{METODOS}

Se trata de una investigación narrativa centrada en el análisis de las respuestas proporcionadas por ocho personas expertas de reconocido prestigio en el campo pedagógico. Esta perspectiva emic nos aproxima a los planteamientos defendidos por los propios participantes. Como ha señalado Harris (1994), permite justificar la validez de los estudios en función del grado de correspondencia existente entre las descripciones proporcionadas por la investigación y la visión de los fenómenos que manifiestan los participantes. Esta posibilidad de penetrar en los otros a través de sus relatos es lo que constituye el eje central en torno al cual gira la investigación narrativa. Para Geertz (1994), este tipo de investigación es una oportunidad para retomar, como foco central de indagación, lo que las personas investigadas dicen de los fenómenos sociales y no sólo lo que puedan decir los investigadores, dando así legitimidad a sus auténticas voces y ofreciendo la oportunidad de ordenar la experiencia y construir la realidad.

La selección de los participantes en este estudio se realizó de acuerdo con los siguientes criterios: 1) personal experto de distintas universidades españolas; 2) profesorado universitario permanente (Catedráticos y Titulares de Universidad); 3) con investigaciones y publicaciones en los últimos años sobre el enfoque de competencias en la educación universitaria y 4) con distintos posicionamientos ante el enfoque de competencias.

Una vez definidos los criterios de selección, se procedió a la elección de los participantes. Para ello, el equipo de investigación, compuesto por profesorado de Facultades de Educación de distintas universidades españolas, elaboró un primer listado de profesionales que fue completado y contrastado con dos bases de datos que recogen, entre otras, las aportaciones del personal investigador español (Dialnet y Google Académico). Posteriormente, se contactó telefónicamente con las personas que formaban parte del este segundo listado para solicitar su colaboración. De este proceso se obtuvo la aceptación de 11 personas a las que se les remitió (vía correo electrónico) el instrumento para la recogida de información. Finalmente, fueron ocho los profesionales participantes, cinco catedráticos y tres titulares en las áreas de Didáctica y Organización Educativa, Didáctica Específicas y Métodos de Investigación y Diagnóstico en Educación. Las personas expertas consultadas pertenecen a la Universidad Autónoma de Barcelona, de Cádiz, de Barcelona, de Deusto, de Granada, de Murcia y Universidad de Valladolid.

En cuanto al instrumento de recogida de la información, un cuestionario compuesto por cuatro preguntas abiertas, fue validado por los miembros del equipo de investigación y por tres personas expertas en métodos de investigación a partir de un protocolo que valoraba la claridad y pertinencia de las preguntas. Las cuestiones definitivas fueron:

1.- ¿Considera usted que el enfoque competencial de la enseñanza superior es positivo para la formación universitaria en general ¿En qué sentido?

2.- ¿Qué aspectos negativos, dificultades o peligros entraña, en su opinión, el enfoque basado en el dominio de competencias adoptado en la Educación Superior?

3.- ¿Considera que el sistema de evaluación de competencias puede mejorar la calidad de la formación que se ofrece en la Universidad?

4.- ¿Qué instrumentos, actividades o estrategias se deben emplear para evaluar las competencias? ¿Podría relacionar las distintas estrategias e instrumentos con los diferentes tipos de competencias y decir cuáles son los más adecuados en cada caso?

\section{RESULTADOS Y DISCUSIÓN}

A continuación mostramos las aportaciones de los participantes en esta investigación en función de las preguntas planteadas y sus puntos de convergencia o divergencia con diferentes aportaciones teóricas relativas al enfoque por competencias. 
De forma general, y en respuesta a la primera pregunta, existe consenso en considerar que el enfoque competencial de la enseñanza superior es positivo para la formación. El profesorado participante lo considera un interesante revulsivo para replantearse el sentido y finalidad de la educación universitaria y para adoptar un nuevo paradigma que confiera mayor protagonismo a los estudiantes en el proceso formativo. Esta idea queda reflejada en las siguientes afirmaciones: "Interesante para la reflexión sobre las finalidades de la enseñanza universitaria" Experto 6 (E6). "Nuevo enfoque de la educación centrada en el aprendizaje de los estudiantes" E1. Algunos de ellos (E4 y E7) reconocen abiertamente el abuso que se ha hecho, en la enseñanza universitaria, del enfoque academicista ("La formación era demasiado académica, con lagunas prácticas" E4) y la necesidad de abandonar la lógica disciplinar que ha imperado en el pasado ("Las disciplinas no son suficientes para aprender competencias profesionales" E7).

Asimismo, el personal experto coincide en que la adopción del nuevo enfoque comporta cambios importantes en la docencia universitaria. El experto 1 alude expresamente a la renovación didáctica de la práctica docente ("Supone una renovación didáctica de la enseñanza universitaria y de la evaluación"). Esta renovación conlleva la revalorización de la formación práctica de los futuros profesionales ("Favorece el desarrollo profesional y mayor preparación para el desempeño profesional y cívico. Favorece aprendizajes con mayor sentido para estudiantes. Les obliga a aprender actuando o haciendo" E3) y su aproximación a la realidad profesional ("El enfoque competencial ayuda a que las carreras universitarias se acerquen más a las demandas del mercado laboral' E8). Para los expertos 2 y 7, el enfoque competencial debe ser, ante todo, una oportunidad para impulsar un debate público en torno a los aprendizajes intelectuales de alto nivel. "Debería ser una ocasión para reflexionar, debatir y concertar entre profesores, alumnos y titulaciones, las operaciones intelectuales complejas como: comprensión, razonamiento, argumentación, resolución de problemas, aprender a aprender,..." E2. El experto 7 considera que fomentar este tipo de aprendizajes "Implica planteamientos metodológicos y organizativos múltiples, abiertos y flexibles" y que una de sus mayores virtualidades es la posibilidad de "Desarrollar competencias profesionales a partir de situaciones y problemas reales".

De acuerdo con estos resultados, podemos señalar que el personal experto del estudio reconoce que el enfoque competencial representa una oportunidad para superar el planteamiento excesivamente academicista de la enseñanza universitaria, ensalzar el valor formativo de la práctica y reforzar la conexión con la realidad profesional, virtualidades también destacadas por Tejada (2006) y Taylor y Hooley (2014). Asimismo, consideran que la lógica disciplinar y la fragmentación por materias representan un serio revés para el desarrollo de competencias, pues su naturaleza integral requiere propuestas formativas interdisciplinares, fruto del trabajo en equipo del profesorado y del apoyo de la institución universitaria, como sugieren Rué y Lodeiro (2010) y Agudo et al. (2013).

Señalan, como positivo, el protagonismo conferido al alumnado en el proceso de aprendizaje y la renovación que, a nivel didáctico, comporta para la enseñanza universitaria. Respecto a este último aspecto, subrayan la incorporación de metodologías activas y sistemas de evaluación alternativos como los cambios más significativos. Elementos estos que han sido igualmente destacados por Bélair et al. (2007).

En cuanto a los aspectos negativos, dificultades o peligros que entraña el enfoque basado en el dominio de competencias (segunda pregunta planteada), la mayor parte de los profesionales que han participado en el estudio muestra su inquietud por el excesivo valor conferido a los resultados de aprendizaje (competencias profesionales), en detrimento de una perspectiva más procesual que englobe otros aprendizajes: "Pone el acento en los resultados dejando de lado el modelo de proceso. Se ha vaciado la formación de la sustancia cultural necesaria" E2; "Puede suponer una mirada restrictiva y desviada si se olvida la atención a los procesos.... Peligro: pensar sólo en competencias profesionales... " E6; "aplicación de un concepto restringido de competencia que sólo considera las específicas de la actividad profesional, sin tener en cuenta las vinculadas con la cultura profesional o su papel en la sociedad actual" E8.

Otra de las preocupaciones más destacada por el profesorado participante está relacionada con el carácter formalista y tecnológico al que se vincula el enfoque competencial, lo que para algunos representa una vuelta al pasado ("...es como volver a la vieja taxonomía de Bloom...listado de competencias sin contenido, mera formalidad... Tal como se ha enfocado implica una mentalidad tecno-burocrática, taxonomía formalista, fragmentación de la formación, poner el acento en los resultados,..." E2). Este excesivo formalismo hace igualmente difícil su articulación y traslación a la práctica docente, ya que no sólo se requiere tiempo y recursos, sino también una adecuada formación ("objeciones prácticas-técnicas asociadas a su formulación e implantación, costo en tiempo, dinero y formación. Transformar los currículos en términos de resultados es una empresa de gran envergadura" E5; "Los cambios de denominación en los títulos que, aun concretando competencias, NO se traducen en contenidos y actividades coherentes con la filosofía" E8). 
La formación y compromiso del profesorado es otro de los elementos clave a considerar: "la aplicación sigue dependiendo de los profesores. Sin ellos nada funciona" E4. El personal experto reconoce, la falta de reflexión y análisis en torno a lo que implica la adopción e implementación del enfoque competencial para la práctica formativa ("No se plantea el fondo de la cuestión, ni la manera en que se utilizan las competencias en el diseño y desarrollo del proceso de e/a (enseñanza-aprendizaje)" E3; "No se plantean, ni entra en el cómo, en la aplicación de esos principios, ni en los pros y contras de las directrices formuladas desde la administración" E7 y "La cuestión es valorar no tanto la filosofía como la aplicación práctica del enfoque. Qué se hace en universidades y carreras" E8.

Según esto, entre los aspectos negativos, los participantes del estudio subrayan el excesivo valor conferido a los resultados de aprendizaje, concretamente a las competencias profesionales. Al igual que Barnett (2001), se muestran críticos con los planteamientos que se decantan por los logros, temen que la adopción del enfoque sea una vuelta a viejos planteamientos tecnológicos, ampliamente cuestionados, y una desconsideración a las posiciones que enfatizan la naturaleza procesual e integral de la formación. Consideran que la formación no se puede reducir a resultados y que éstos son difícilmente medibles a corto plazo. Denuncian la relevancia conferida a las demandas del mercado y al "saber hacer", en detrimento del "saber" y del "ser", y apuestan por una formación universitaria que desarrolle competencias profesionales, pero que también forme buenos ciudadanos en la esfera personal, cultural y social, y no exclusivamente profesional. Los participantes en la investigación abogan por una postura que complemente las dos lógicas subrayadas por Escudero (2009) - humanista vs mercantilista-, asumiendo así que, desde una perspectiva compleja de la realidad, es posible la complementariedad entre posicionamientos aparentemente antagónicos (Morin, 2000), posición esta que compartimos plenamente.

También para Perrenoud (2008) la disyuntiva entre el saber y la competencia es injustificada, puesto que el desarrollo de competencias no implica relegar a los saberes, sino dotarles de una fuerza nueva que les vincula a las prácticas sociales y a la formación de competencias indispensables para la vida, competencias que, en su opinión, abren nuevas oportunidades a mayor número de ciudadanos. Además, esta escisión contraviene peligrosamente los pilares en que, según la Comisión Internacional sobre la educación para el siglo XXI (Delors, 1996), debe sustentarse la educación: aprender a conocer, aprender a hacer, aprender a vivir juntos y aprender a ser. Quizá por ello proponen que la formación universitaria se aborde desde planteamientos más integradores, menos reduccionistas, que englobe la formación para el mercado laboral, pero que también la trascienda.

Por otra parte, los participantes del estudio reconocen que el excesivo formalismo que lleva asociado el diseño y trabajo por competencias representa un obstáculo para el éxito del cambio. Comparten, así, la posición de Fullan (2003: 297) cuando afirma que: "la planificación técnica no ha dado resultado en el cambio educativo". Factores como estos generan, en opinión de los participantes en el estudio expertos, escisiones entre la filosofía y propósitos perseguidos (lo que se formaliza en el diseño) y lo que realmente se hace en la práctica. Estos temores quedan, en parte, ratificados en los resultados de algunas investigaciones (Agudo, et al., 2013; Ion y Cano, 2012). En la indagación llevada a cabo por estas últimas autoras, por ejemplo, ya se señalaba que sólo el $27,6 \%$ de profesorado universitario afirma evaluar competencias, a pesar de existir un compromiso explícito en el diseño de las nuevas titulaciones que recoge la obligación de hacerlo. También en el contexto internacional se duda de que la adopción del enfoque por competencias represente una verdadera transformación de la práctica. Así lo recogen Marín et al. $(2013,45)$ en la siguiente reflexión: "hasta qué punto el concepto de competencia se ha convertido o se puede convertir en retórica y en adorno textual de los mejores discursos y documentos académicos; o hasta dónde podemos trascender del momento conceptual a expresiones de práctica educativa transformada y transformadora"

Centrándonos en la opinión sobre si el sistema de evaluación de competencias puede mejorar la calidad de la formación que se ofrece en la Universidad (pregunta 3), en general, las respuestas a esta pregunta han sido afirmativas, considerando que "El sistema de evaluación es fundamental en el desarrollo de competencias y en la calidad de la formación" E3, aunque con condicionantes.

Un primer grupo de condiciones está relacionado con el propio sistema de evaluación de competencias. En este sentido, la evaluación de competencias se considera un factor clave de mejora de la formación si ésta no sólo se utiliza para calificar: "depende de qué sistema de evaluación. Si sólo calificamos se produce poca mejora y nula formación" E4, si se apoya en una adecuada selección de competencias: "Se dé una adecuada valoración y validación de las competencias o logros de aprendizaje" (E5), si previamente se han llevado a cabo tareas de aprendizaje dirigidas a la adquisición de competencias (E5): “(...) que los estudiantes realicen tareas pertinentes en relación a los logros a alcanzar", y si se rompe la planificación ceñida a asignaturas compartimentadas (...) que se de una organización de la enseñanza que rompa el MICROSISTEMA de las asignaturas" (E5). Además, en opinión del personal experto, esta evaluación debe favorecer la reflexión sobre la práctica a fin de desvelar los dilemas y disonancias implícitos en ella "La realización práctica de la 
evaluación ayuda a comprender la complejidad de la propia formación y las incoherencias que debemos evitar" E8. La evaluación se convierte así en instrumento de formación y de práctica docente reflexiva.

Otro de los condicionantes estaría relacionado con el funcionamiento de este tipo de evaluación. El experto 2 resalta que habría que estudiar y documentar cómo está funcionando el sistema de evaluación por competencias, ya que, a su entender, "faltan investigaciones específicas que describan, analicen e interpreten cómo se están utilizando las competencias y qué se está evaluando” E2, lo que dificulta cualquier valoración sobre su impacto y cualquier intento de mejora. Los participantes en el estudio coinciden, por tanto, en considerar al profesorado un factor clave del cambio, pero reconocen que existe poca reflexión, debate y valoración sobre lo que realmente supone la adopción e implementación del enfoque por competencias profesionales. Esta falta de reflexión obstaculiza, en su opinión, el enfoque competencial y su correcta articulación en la práctica formativa y de evaluación, corriendo el riesgo de que este quede en un mero ejercicio intelectual baladí como advierten Marín et al. (2013).

Otros factores a considerar son la necesidad de formación del profesorado y de coordinación académica. El experto 6 insiste en la "necesidad de la coordinación de equipos docentes en la discusión y reflexión sobre el proceso y las finalidades de la formación universitaria. Puesto que la evaluación es CLAVE y condiciona todo el proceso de e/a (enseñanza-aprendizaje)" E6. Termina diciendo que "la evaluación por competencias tiene un valor per se....y como desencadenante de una reflexión y de un proceso de trabajo docente" E6. La necesidad de una adecuada formación docente es corroborada por el experto 7 cuando se pregunta: “ ¿tenemos la competencia profesional los docentes para afrontar una evaluación de esta naturaleza?” E7. En este sentido, este mismo experto se muestra pesimista por varios motivos: excesiva dotación docente, escasa formación del profesorado, falta de cultura evaluativa y recursos y escaso apoyo económico. Situaciones estas que evidencian las condiciones poco favorables en que se ejerce la docencia universitaria y que, en los últimos años, han intensificado su deterioro debido a la crisis económica y a los consiguientes recortes aplicados a la educación universitaria.

Finalmente, en la cuarta pregunta, el profesorado experto, no sólo ha aludido a los instrumentos, actividades o estrategias que deben emplearse para evaluar competencias, sino que sus respuestas reflejan su concepción sobre la propia evaluación de competencias y sus características. La mayoría opina que "Implica un enfoque renovado de la evaluación" E1 y apuesta por una evaluación auténtica "Se producen situaciones múltiples, auténticas. Hacia la evaluación auténtica” E1. Consideran, igualmente, que se adecúa mejor a la naturaleza del proceso enseñanza-aprendizaje, ya que "Los esquemas de evaluación se han de aplicar con carácter gradual, es decir captar cómo van siendo los contenidos y procesos que van componiendo el desarrollo del aprendizaje" E2. El experto 7, considera que la evaluación de competencias debe "evaluar el aprendizaje de los saberes" E7 y permitir la posibilidad de "tender a la AUTOREGULACION del proceso de aprendizaje por parte de los alumnos (feedback)" E6. Se enfatiza así la apuesta de los participantes por una evaluación de procesos que englobe también a los saberes y enfatice el valor formativo de la retroalimentación.

A la hora de relacionar los instrumentos de evaluación más adecuados con las competencias, la mayor parte de los participantes destaca la dificultad de abordar esta tarea y lo inadecuado de establecer un listado de instrumentos desconectados de las tareas y los contextos concretos. Así lo corroboran los profesionales 4, 6 y 7: "Hay que encontrar (usar) el instrumento más adecuado a cada competencia en situaciones reales y contextos reales" E4, "se han de diseñar situaciones de aprendizaje parecidas a las reales" E6 y "Hay que buscar /usar dispositivos válidos y fiables que produzcan evaluaciones, aunque no se debe olvidar que las competencias se ven en la ACCION y se evalúan" E7.

Un principio importante es la pluralidad y triangulación de los instrumentos. Los participantes del estudio consideran necesario diversificar los instrumentos de evaluación y opinan que la pertinencia del instrumento no viene determinada por el instrumento en sí, sino por el tipo de tarea a evaluar y la finalidad que se persigue "Hay que relacionar las competencias a desarrollar con la TAREA a ejecutar" E5, de tal forma que "Todas las estrategias e instrumentos de evaluación pueden ser válidos ligados a la validez de la tarea” E5. Por tanto, "Más importante que los instrumentos que se utilizan es CÓMO se utilizan" E4, y cuál es el fin que se persigue con su uso: “¿Para calificar, formar, informar al alumno para su mejora?” E4.

A pesar de la dificultad de establecer un listado de instrumentos, el personal experto se muestra concluyente al considerar que los exámenes teóricos no son útiles para evaluar competencias ("El examen teórico no sirve para evaluar las competencias” E4) y señalan, como adecuados, los siguientes: portafolios, ensayos e informes, estudios de caso, resolución problemas, escalas, parrillas, rúbricas, debates, pruebas reflexivas, listas de control, exposiciones orales; ,instrumentos, muchos de ellos, ya señalados, como pertinentes, por otros autores (Brown y Glasner, 2010; Marín et al., 2013). 
En opinión de la experta 5, la evaluación debe combinar pruebas teórico-prácticas y sugiere: exámenes, entrevistas, portafolios, proyectos, role-playing, escalas de observación y rúbricas. Sólo el experto 3 presenta una breve relación de competencias y cómo evaluarlas, que mostramos en la Tabla 1.

Tabla 1: Competencias y procedimientos de evaluación

\begin{tabular}{|l|l|}
\hline Trabajo en equipo & Evaluación entre compañeros \\
\hline Comunicación oral & Presentaciones orales \\
\hline Comunicación escrita & Trabajos escritos \\
\hline Ética & Dilemas, casos, debates \\
\hline Aprender a aprender & $\begin{array}{l}\text { Reflexión sobre el proceso de e/a (enseñanza- } \\
\text { aprendizaje). }\end{array}$ \\
\hline
\end{tabular}

Además de detallar el listado de instrumentos, los participantes los clasifican en función de la tradición ("la evaluación clásica" E5, "tradicional con papel y lápiz en conexión con el ámbito cognoscitivo" E7) y según modalidades de evaluación alternativas. En este caso hay coincidencia en considerar el portafolios, la autoevaluación y la evaluación entre iguales, como opciones útiles para evaluar competencias. Se aprecian, no obstante, algunas diferencias en cuanto a la ubicación de algunos de los instrumentos o estrategias señalados. Así, la experta 5, cuando presenta los instrumentos o estrategias que se emplean en la evaluación clásica, incluye: "pruebas objetivas, ensayos, resolución de problemas, casos, trabajos de investigación, ejecuciones prácticas: observación in situ o en diferido" E5, y en las nuevas formas de evaluar señala: "evaluación basada en logros, portafolios, autoevaluación y evaluación entre iguales" E5; mientras que el experto 7 incluye, dentro de la evaluación alternativa, "las evaluaciones de ejecución, autoevaluación, diario, evaluación por colegas, $360^{\circ}$ y balance y auto balance de competencias" E7. Este mismo experto incorpora también la "resolución de problemas y prácticas profesionales", instrumentos estos que la experta 5 habían incluidos dentro de la evaluación clásica. Obsérvese que, en ambos casos, más que presentar instrumentos concretos aluden a procedimientos y modalidades de evaluación.

Esta falta de convergencia en la clasificación de los instrumentos y el hecho de que no se distinga entre instrumentos, procedimientos y modalidades de evaluación, pone de manifiesto la falta de acuerdo y reflexión en torno a este aspecto entre el personal experto en pedagogía

En este sentido, nos permitimos subrayar algunos aspectos referenciados que podrían generar cierta controversia. En primer lugar, el hecho de que la evaluación basada en logros se presente como evaluación alternativa para evaluar competencias no aclara si esos logros aluden sólo a competencias profesionales, o si se contemplan otros aprendizajes de naturaleza más integral, posibilidad ésta que sería deseable si se apuesta por un modelo de competencias más holístico.

Por otro lado, en la medida en que la evaluación de competencias requiere una descomposición en comportamientos o competencias más simples y del establecimiento de niveles de logro o ejecución, se corre el riesgo de abocar el enfoque por competencias a una planificación por objetivos que recuerda la vieja planificación tecnológica. En tercer lugar, la falta de una cultura colaborativa y de coordinación docente en la universidad podría dejar en manos del profesorado, a título individual, estas decisiones, lo cual no sería deseable. Aspectos como éstos, hacen aconsejable ampliar el debate en torno a la evaluación de competencias en el ámbito universitario.

Entre el personal experto también hay quien distingue entre aquellos instrumentos que son más útiles para evaluar las competencias básicas y aquellos otros que lo son para las competencias transversales y específicas. Así, la evaluación de las competencias básicas, en su opinión, ha de estar vinculada a las prácticas profesionales: "La evaluación de las competencias básicas se ha de vincular mucho a las prácticas profesionales (aunque se trabajan e incorporan a todas las materias y situaciones)" E8 y los instrumentos más adecuados son el informe de tutores y el autoinforme. En el caso de las competencias transversales, considera que deben estar ligadas a actividades evaluativas relacionadas con las prácticas profesionales y al proyecto de fin de carrera, ya "que debe de hacerse desde las actuaciones globales, que permiten valorar el dominio final de las competencias pretendida" E8. Finalmente, para las competencias específicas, propone instrumentos de evaluación "que tengan que ver con la evaluación de procesos (portafolios, pruebas reflexivas, listados de control, exposiciones orales, informes de autoevaluación, seguimiento de grupos de discusión,...) y con los productos (actividades de aplicación, pruebas de verificación, resolución de casos...), en función de la carrera y cursos considerados" E8.

En consonancia con la posición expresada por las personas expertas, que aboga por una formación universitaria que conjugue el desarrollo de competencias profesionales con una formación más holística que 
atienda al desarrollo personal y el ejercicio de la ciudadanía en una sociedad democrática y solidaria, estos participantes apuestan por una evaluación integral que, apoyándose en una diversidad de instrumentos, incluya a la evaluación de competencias profesionales sin olvidar las competencias básicas, reivindicando así, la posibilidad de salvar las distancias entre las dos lógicas señaladas por Escudero (2009) y los dos discursos sobre competencias que recoge Bolívar (2011). Estos discursos, como señala este último autor, tienen distinto origen y responden a diferentes propósitos. El dirigido a la definición de las competencias en Educación Básica, vinculado al Proyecto DeSeco, está más comprometido con garantizar la equidad y la ciudadanía, mientras que el centrado en la definición de perfiles profesionales, cercano al proyecto Tuning, prima las demandas del mercado laboral. En este sentido, valoramos positivamente las iniciativas integradoras emprendidas a nivel universitario para salvar esta escisión (Schmal, 2015) y que, en nuestra opinión, representan una clara oportunidad, no sólo para romper con la dicotomía entre ambos discursos, sino para hacer frente a las presiones que, desde la corriente neoliberal, se ejercen hacia la educación superior y que pretenden convertirla en un instrumento al servicio de sus intereses.

El personal experto se muestra convencido de que el enfoque competencial conlleva, necesariamente, un cambio del sistema, estrategias e instrumentos de evaluación. Consideran que se ha de buscar la coherencia entre el diseño por competencias, el proceso de aplicación y la evaluación, ya que, como afirman García y Morillas (2011: 119) en referencia a esta última: "Ésta no constituye una tarea a realizar al margen de los procesos educativos, sino que es una actividad integrada en la misma acción docente que se lleva a cabo". Se trata de conseguir una evaluación "auténtica" (Arraiz y Sabiron, 2009) que valore no sólo el saber (conocimiento), sino el resto de los saberes (el saber cómo, demostrar cómo y hacer). La evaluación debe ser integral, de tal modo que abarque diferentes tipos de aprendizaje.

Otra característica de la evaluación competencial, en opinión de los participantes, es que posibilita la autorregulación del alumnado en el proceso de aprendizaje. Idea ya subrayada por Edwards y Usher (1994) en su trabajo Disciplining the subject: the power of competence, en el que destaca que las prácticas de evaluación competencial no sólo ejercen una influencia directa sobre los estudiantes, sino que promueven formas de auto-vigilancia que los disciplina a través de su propio auto-monitoreo sin que ellos sean conscientes de lo que está ocurriendo (Boud, Cohen y Sampson, 2001). Opinión esta que también han ratificado recientemente Agudo et al. (2013).

El enfoque de competencias es visto como el sistema que más se adecúa al propio proceso de aprendizaje por ser ambos graduales. Este carácter gradual en la adquisición de la competencia, destacado por uno de los participantes, es coherente con la gradación propuesta por Jornet et al (2011) para evaluar la adquisición de competencias.

La mayor parte de los profesionales del estudio opina que el valor y pertinencia de las estrategias e instrumentos de evaluación vienen determinados por la competencia que se pretende desarrollar y la situación, real o simulada, en que se usa. Esta apreciación, ha sido también destacada por Nicol (2007: 677) en las conclusiones de su trabajo cuando afirma: "Una observación interesante de uno de estos estudios de caso es el papel de las pruebas de opción múltiple objetivas [...] no es la prueba en sí que es importante, pero si el contexto de su uso".

Los participantes expertos se muestran igualmente partidarios de emplear diversos y variados instrumentos de evaluación para determinar el grado de adquisición/desarrollo de las competencias, opinión esta compartida por Baartma et al. (2007) y García y Morillas (2011). Estos últimos afirman que: "hay que conocer y utilizar variedad de instrumentos de recogida de información, establecer los criterios de calidad para cada uno, así como su ponderación, de manera que los estudiantes sepan en base a qué se les va a evaluar y el peso de cada procedimiento en función de las competencias adquiridas" (García y Morillas, 2011: 123). Desde esta posición, coinciden en destacar el portafolios, la autoevaluación y la evaluación entre iguales, como las opciones más útiles en la evaluación de competencias. Instrumentos estos que también han sido sugeridos, entre otros, por Brown y Glasner (2010) y Agudo et al. (2013)

En opinión de las personas expertas del estudio, el sistema de evaluación de competencias puede ayudar a mejorar la calidad de la formación que ofrece la Universidad si se lleva a cabo con coherencia, mejoran las condiciones en las que se aplica y existe una adecuada formación del profesorado.

Por último, la calidad de la propia evaluación, así como la necesidad de un conocimiento riguroso de cómo está funcionando el supuesto sistema de evaluación por competencias han sido destacadas también por Baartman et al. (2007) y Arraiz y Sabirón (2010) como condicionantes para la mejora. Estos últimos, después de analizar distintos decálogos sobre buenas prácticas evaluativas, establecen cinco características básicas para definir una evaluación de calidad o auténtica: utilizable, rentable, razonablemente exacta y con resultados verídicos que ayuden en la toma de decisiones y sirva de reconocimiento y recompensa, que 
proporcione a los estudiantes normas claras para alcanzar resultados aceptables. Sin embargo, Baartman et al. (2007) recogen en su estudio la opinión del profesorado sobre los criterios de calidad de la evaluación de competencias y concluyen que, tanto los criterios de calidad clásicos (comparabilidad, equidad, reproducibilidad de las decisiones y transparencia), como los basados en competencias (autenticidad/realismo, complejidad cognitiva, coste y eficiencia, inmediatez, consecuencias educativa y significatividad) son igualmente importantes, reivindicando así, la necesidad de un modelo integrador de evaluación más coherente con la visión holística de competencia que demandan los profesionales de pedagogía que han participado en el estudio.

\section{CONCLUSIONES}

Los resultados recabados en la investigación nos permiten establecer las siguientes conclusiones:

1. Los profesionales expertos del estudio se muestran críticos ante el enfoque por competencias, pues señalan aspectos positivos, pero también reconocen algunos peligros. Respecto a los primeros, coincide en que el enfoque por competencias es una oportunidad para redefinir el sentido y finalidad de la educación universitaria, reforzar su compromiso con aprendizajes más ligados a la práctica profesional, y dotar al alumnado de un mayor protagonismo en los procesos de formación. Sin embargo, cuestionan el excesivo valor conferido a los resultados de aprendizaje y el hecho de que éstos se definan, exclusivamente, en términos de competencias profesionales. Consideran que la institución universitaria debe adoptar perspectivas más procesuales y holísticas de los procesos formativos, cultivar aprendizajes más integrales y vitales que incorporen las competencias profesionales pero que también las trasciendan, permitiendo a las personas vivir plenamente su ciudadanía. Se muestran convencidos, de que la universidad no puede limitarse a atender exclusivamente las demandas laborales, ni regirse por la lógica de los mercados.

2. En opinión de los profesionales de la Pedagogía que participan en el estudio, el enfoque por competencias podría ayudar a instaurar una nueva cultura docente en la universidad basada en la colaboración, pero para ello sería necesario, en su opinión, superar la lógica disciplinar y academicista que tradicionalmente ha primado en la institución universitaria.

3. Los participantes en la investigación recelan del carácter excesivamente formalista que comporta el enfoque competencial y que algunos asocian al enfoque tecnológico de enseñanza. Consideran que este o formalismo obstaculiza las posibilidades reales de renovación y mejora de la práctica educativa en la universidad. Temen que las mejoras que podría aportar el enfoque por competencias a la enseñanza universitaria se queden, como en tantas otras reformas educativas, en un mero discurso entre expertos y administradores que poco 0 nada contribuye a transformar la práctica educativa. Esta desconfianza hacia el poder renovador que podría tener el enfoque por competencias en la enseñanza universitaria se acentúa cuando aluden al profesorado. En este caso, se muestran tajantes al reconocer que la escasa formación docente del profesorado universitario y las condiciones poco favorables en que ejercen la docencia (devaluación de la enseñanza, masificación, excesiva dotación docente, falta de cultura colaborativa y evaluativa en la enseñanza, escasez de recursos y apoyo económico ...) no favorecen su implicación en el proceso de cambio, a pesar de ser ésta una condición imprescindible para mejorar la enseñanza y favorecer el éxito de cualquier reforma educativa. Reconocen, así, que ha sido un cambio gestado al margen del profesorado. En general, el personal experto que ha participado en la investigación cree, firmemente, que en la introducción del enfoque por competencias en la universidad ha faltado debate, reflexión y análisis sobre lo que realmente implica.

4. Los profesionales expertos del estudio opinan que el sistema de evaluación de competencias puede mejorar la calidad de la formación universitaria si se realiza una adecuada valoración y validación de las competencias, si las tareas que se plantean son coherentes con los logros que se persiguen, y si se concibe la evaluación como un instrumento de formación y mejora. Reconocen, en este sentido, que se adecúa mejor a la naturaleza del proceso enseñanza-aprendizaje y valoran positivamente que favorezca la autorregulación del alumnado en su aprendizaje.

5. Los participantes en la investigación creen necesario diversificar los instrumentos de evaluación y opinan que su pertinencia está en función del tipo de tarea a evaluar, la finalidad que se persigue y de cómo se usen en un contexto concreto. Por ello, se muestran reticentes a vincular instrumentos de evaluación a competencias concretas, ya que entienden que la competencia está asociada al desempeño de una acción en un contexto específico que determina la pertinencia e idoneidad del propio instrumento de evaluación, por lo que resulta complejo establecer cualquier vinculación de antemano. Subrayan, así, la estrecha relación existente entre las competencias y los contextos donde se adquieren y aplican.

6. Existe un amplio acuerdo entre el personal experto en considerar que los exámenes teóricos no son útiles para evaluar competencias y se pronuncian a favor de otras opciones alternativas como: portafolios, autoevaluación y evaluación entre iguales y, en menor medida, estudio de caso, resolución de problemas, escalas y rúbricas. 
7. Finalmente, los profesionales de la Pedagogía que han participado en la investigación consideran que los estudios realizados hasta el momento sobre evaluación de competencias son insuficientes. Reconocen que se sabe poco sobre qué se está evaluando, cómo se está realizando esa evaluación o cuál es el impacto en el desarrollo competencial o en la mejora de la formación universitaria. Se asume así, que la investigación en evaluación de competencias es un aspecto poco explorado en este proceso de cambio a pesar de ser un factor clave.

Estas conclusiones abren nuevos debates y líneas de trabajo sobre el enfoque de competencia en el ámbito universitario y sobre el papel que juega la evaluación en el desarrollo de las mismas. Líneas que podrían desarrollarse con otros instrumentos de investigación, como la entrevista o los grupos de discusión, que permitirían el acceso a una información más rica que la aportada por el cuestionario abierto que se ha aplicado en este estudio. Para avanzar en este sentido, consideramos necesario poner en marcha iniciativas de investigación y de acción que impliquen a instituciones y docentes en la búsqueda de una tercera lógica que permita complementar el discurso que subraya el valor de la educación en la sociedad del conocimiento y el compromiso de la universidad con una educación de calidad, equitativa y justa, con aquel otro que reconoce el valor de la educación para promover el desarrollo económico, la funcionalidad de los aprendizajes y la empleabilidad.

\section{REFERENCIAS}

Agudo, J.E., R. Hernández-Linares, M. Rico y H. Sánchez, Competencias Transversales: Percepción de su Desarrollo en Ingeniería en Diseño Industrial y Desarrollo de Productos, doi:10.4067/S071850062013000500006, Formación Universitaria, (en línea), 6(5), 39-50 (2013)

Arraiz, A. y F. Sabirón, La Evaluación como Herramienta de Aprendizaje: Decálogo Fundamentado y Ejemplificado para una Evaluación Auténtica. Universidad de Zaragoza- Fondo Social Europeo, Zaragoza, España (2010)

Baartman, L. K. J., T.J. Bastiaens, P.A. Kirschner, C.P.M. y van der Vleuten, Teachers' Opinions on Quality Criteria for Competency Assessment Programs, Teaching and Teacher Education: An International Journal of Research and Studies, 23 (6), 857-867 (2007)

Barnett, R., Los Límites de la Competencia: el Conocimiento, la Educación Superior y la Universidad, Gedisa Madrid, España (2001)

Bélair, L., D. Laveault, y C. Lebel, Les Compétences Professionnelles en Enseignement et Leur Évaluation. Presses de l'Université d'Otawa, Otawa, Canadá (2007)

Bolívar, A., El Enfoque por Competencias en Educación General y Educación Superior: entre la Inclusión Social y el Mercado Laboral, en Reforma Universitária. E A Construção do Espaço Europeu de Educação Superior. Análise de uma Década do Processo de Bolonha por E. Monteiro de Aguiar y M.L. Pinto de Almeida (Orgs.), pp 109-156 Mercado de Letras, Campinas, Brasil (2011)

Boud, D., R. Cohen y J. Sampson, Peer Learning and Assessment, Assessment and Evaluation in Higher Education, 24 (4), 413-426 (2001)

Brown, S. y A. Glasner (Edit.), Evaluar en la Universidad. Problemas y Nuevos Enfoques, Narcea, Madrid, España (2010)

Coll, C., Las Competencias en la Educación Escolar: Algo Más que una Moda y Mucho Menos que un Remedio, Aula de innovación educativa, 16 (1), 24-39 (2007)

Delors, J., La Educación Encierra un Tesoro, Santillana Ediciones Unesco, Madrid, Madrid (1996)

Escudero, J.M., Las Competencias Profesionales y la Formación Universitaria: Posibilidades y Riesgos, Red U. Revista Interuniversitaria de Pedagogía Social, 16, 65-82 (2009)

Fullan, M., Emoción y Esperanza: Conceptos Constructivos para Tiempos Complejos, en Replantear el Cambio Educativo. Un Enfoque Renovador por A. Hargreaves (Comp.), pp. 296-317 Amorrortu Editores, Madrid, España (2003)

García, M.P. y L.R. Morillas, La Planificación de Evaluación de Competencias en Educación Superior, Revista Electrónica Interuniversitaria de Formación del Profesorado, ISSN 1575-0965, (en línea; 
http://www.aufop.com/aufop/uploaded_files/articulos/1302193022.pdf, acceso 21 de octubre 2014), 14(1), 113-124 (2011)

Geertz, C., Conocimiento Local. Ensayos sobre la Interpretación de las Culturas, Paidós, Barcelona, España (1994)

Halbaut, L.; E. García y M. Aróztegui, Metodologías para el Desarrollo de Competencias, en Formación y Evaluación por Competencias en Educación Superior por G. Londoño y E. Cano (Edits.), pp 67-89 Ediciones Unisalle, Bogotá, Colombia (2015)

Harris, M., Introducción a la antropología general, Alianza, Madrid, España (1994)

Ion, G. y E. Cano, La Formación del Profesorado Universitario para la Implementación de la Evaluación por Competencias, Educación XX1, 15 (2), 249-270 (2012)

Jackson, D., Business Undergraduates' Perceptions of their Capabilities in Employability Skills: Implications for Industry and Higher Education, Industry and Higher Education, 26(5), 345-356 (2012)

Jornet, J.M., J. González, J.M. Suárez y M.J. Perales, Diseño de Procesos de Evaluación de Competencias: Consideraciones acerca de los Estándares en el Dominio de las Competencias, Bordón, 63 (1), 125-145 (2011)

López, M.C., El Proceso de Bolonia: Profesorado y Modelo Educativo Centrado en el Aprendizaje de Competencias, en Reforma Universitária. E A Construção do Espaço Europeu de Educação Superior. Análise de uma Década do Processo de Bolonha por E. Monteiro de Aguiar y M.L. Pinto de Almeida(Orgs.), pp 157180 Mercado de Letras, Campinas, Brasil (2011)

Marín, R., I. Guzmán, A. Márquez y M. Peña, La Evaluación de Competencias Docentes en el Modelo DECA: Anclajes Teóricos, doi: 10.4067/S0718-50062013000600005, Formación Universitaria, (en línea), 6(6), 41-54 (2013)

Morin, E., La Mente Bien Ordenada,Seix Barral, Barcelona, España (2000)

Nicol, D., Laying a Foundation for Lifelong Learning: Case Studies of E-Assessment in Large 1st-Year Classes, British Journal of Educational Tecnology, 38(4), 668-678 (2007)

Palmer, A., J. Montaño y M. Palou, Las Competencias Genéricas en la Educación Superior. Estudios Comparativo entre la Opinión de Empleadores y Académicos, Psicothema, 21(3), 433- 438 (2009)

Parris, M. y K. Saville, Piecing Together the Puzzle of Graduate Employment: Factors that Shape the Graduate Work Expectations of Human Resource Management Students, Industry and Higher Education, 25(1), 15-24 (2011)

Perrenoud, P., Construir las Competencias, ¿Es Darle la Espalda a los Saberes?, Red U. Revista de Docencia Universitaria, 6(2), 1-16 (2008)

Rué, J. y L. Lodeiro, Equipos Docentes y Nuevas Identidades Académicas, Narcea, Madrid, España (2010)

Schmal, R., Evolución de un Programa de Formación en Competencias Genéricas, doi: 10.4067/S071850062015000600012, Formación Universitaria, (en línea) 8(6), 95-106 (2015)

Taylor, A.R. y T. Hooley, Evaluating the Impact of Career Management Skills Module and Internship Programme within a University Business School, British Journal of Guidance \& Counselling, 42(5), 487-499 (2014)

Tejada, J., Elementos de Convergencia entre la Formación Profesional y la Universidad: Implicaciones para la Calidad de la Formación Profesional Superior, Revista de Educación, 340, 1085-1117 (2006) 